\title{
Total Duration of Antimicrobial Therapy in Veterans Hospitalized With Uncomplicated Pneumonia: Results of a National Medication Utilization Evaluation
}

\author{
Karl J. Madaras-Kelly, PharmD, MPH ${ }^{1 \star}$, Muriel Burk, PharmD²,3, Christina Caplinger, PharmD4, Jefferson G. Bohan, PharmD4, \\ Melinda M. Neuhauser, PharmD, MPH³, Matthew Bidwell Goetz, MD5, Rongping Zhang², Francesca E. Cunningham, PharmD²,3; \\ for the Pneumonia Duration of Therapy Medication Utilization Evaluation Group
}

\begin{abstract}
'Pharmacy Service, Veterans Affairs Medical Center and Department of Pharmacy Practice, College of Pharmacy, Idaho State University, Meridian, Idaho; '2Center for Medication Safety, Hines VA, Hines, Illinois; ${ }^{3}$ VA Pharmacy Benefits Management Services, Hines VA, Hines, Illinois; ${ }^{4}$ Pharmacy Service, Veterans Affairs Medical Center, Boise, Idaho; ${ }^{I}$ nfectious Diseases Section, VA Greater Los Angeles Health Care System and David Geffen School of Medicine at University of California-Los Angeles, Los Angeles, California.
\end{abstract}

OBJECTIVE: Practice guidelines recommend the shortest duration of antimicrobial therapy appropriate to treat uncomplicated pneumonia be prescribed to reduce the emergence of resistant pathogens. A national evaluation was conducted to assess the duration of therapy for pneumonia.

DESIGN: Retrospective medication utilization evaluation.

SETTING: Thirty Veterans Affairs medical centers.

PATIENTS: Inpatients discharged with a diagnosis of pneumonia.

MEASUREMENTS: A manual review of electronic medical records of inpatients discharged with uncomplicated community-acquired pneumonia (CAP) or healthcareassociated pneumonia (HCAP) was conducted. Appropriate CAP therapy duration was defined as at least 5 days, and up to 3 additional days beginning the first day the patient achieved clinical stability criteria; the appropriate HCAP therapy duration was defined as 8 days. The duration of antimicrobial therapy for intravenous (IV) and oral (PO) inpatient administration, PO therapy dispensed upon discharge,
Clostridium difficile infection (CDI), hospital readmission, and death rates were measured.

RESULTS: Of 3881 pneumonia admissions, 1739 met inclusion criteria (CAP [ $n=1195]$; HCAP [ $n=544]$ ). Overall, $13.9 \%$ of patients (CAP [6.9\%], HCAP [29.0\%]) received therapy duration consistent with guideline recommendations. The median (interquartile range) days of therapy were 4 days (3-6 days), 1 day (0-3 days), and 6 days (4-8 days) for inpatient IV, inpatient PO, and outpatient PO antimicrobials, respectively. CDI was rare but more common in patients who received therapy duration consistent with guidelines. Therapy duration was not associated with the readmission or mortality rate.

CONCLUSIONS: Antimicrobials were commonly prescribed for a longer duration than guidelines recommend. The majority of excessive therapy was completed upon discharge, identifying the need for strategies to curtail unnecessary use postdischarge. Journal of Hospital Medicine 2016;11:832-839. (c) 2016 Society of Hospital Medicine
Pneumonia is the leading inpatient infectious diagnosis for which antimicrobials are prescribed in the United States. ${ }^{1}$ Supported by moderate- to high-quality evidence, guidelines produced jointly by the Infectious Diseases Society of America (IDSA) and American Thoracic Society (ATS) recommend treating pneumonia with the shortest appropriate duration of antimicrobial therapy to minimize risk for antimicrobial-related adverse events. ${ }^{2-4}$

Evidence supports short duration of therapy for treatment of uncomplicated pneumonia. ${ }^{3-12}$ IDSA/ ATS guidelines state, "patients with CAP [community-

\footnotetext{
*Address for correspondence and reprint requests: Karl J. Madaras-Kelly, Veterans Affairs Medical Center, T111, 500 West Fort Street, Boise, ID 83713; Telephone: 208-422-1000; Fax: 208-422-1155;

E-mail: karl.madaras-kelly2@va.gov

Additional Supporting Information may be found in the online version of this article.

Received: December 23, 2015; Revised: June 23, 2016; Accepted: June 28, 2016

2016 Society of Hospital Medicine DOI 10.1002/jhm.2648

Published online in Wiley Online Library (Wileyonlinelibrary.com).
}

acquired pneumonia] should be treated for a minimum of 5 days (level 1 evidence), should be afebrile for 48-72 hours, and should have no more than 1 CAP-associated sign of clinical instability... .before discontinuation of therapy (level II evidence). (Moderate recommendation.) A longer duration of therapy may be warranted if initial therapy was not active against the identified pathogen or if it was complicated by [abscess, empyema, severe immunosuppression, or] extra-pulmonary infection such as meningitis or endocarditis. (Weak recommendation; level III evidence)."3 Recommended therapy duration for patients with uncomplicated healthcare-associated pneumonia (HCAP) who respond to initial therapy is 7 to 8 days unless gram-negative nonfermenting rods or complications are identified (level I evidence). 4

Within the Veterans Health Administration (VHA), the Antimicrobial Stewardship Taskforce (ASTF) was created to optimize care by developing, deploying, and monitoring a national-level strategic plan for antimicrobial therapy management improvements. ${ }^{13,14}$ Although singlecenter studies have found antimicrobial therapy for CAP 
Admissions meeting pneumonia ICD-9 criteria

identified by VA MedSAFE $(n=3881)$

conducted locally at $\mathbf{3 0}$ VA facilities

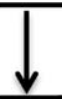

Records Excluded from the Duration of Therapy Analysis*

- Record review did not verify presence of all inclusion criteria ( $n=962)$

- Pneumonia/septicemia diagnosis not documented in discharge summary $(n=327)$

- Patient admitted for $<\mathbf{4 8}$ hours $(n=\mathbf{2 7 5})$

- Patient did not survive until discharge $(n=188)$

- Received antibiotics $>\mathbf{4 8}$ hours after admission or $\leq \mathbf{2 4}$ hours of antibiotics before discharge ( $n=172)$

- Record review identified presence of exclusion criteria $(n=1216)$

- Complicated, immunosuppressed, or extra-pulmonary source ( $n=508)$

- Transferred directly to or from another facility where hospitalization occurred $(n=344)$

- Discharged for pneumonia within $\mathbf{2 8}$ days prior to admission ( $n=156)$

- Full antimicrobial course not completed inpatient AND/OR discharged without documentation of continued outpatient therapy $(n=88)$

- Documentation of pneumonia not present within $<48$ hours of admission ( $n=74$ )

- Length of stay for index admission $>14$ days $(n=46)$

* Patients may have met more than one criteria resulting in exclusion.

FIG. 1. Application of inclusion and exclusion criteria for the pneumonia duration of therapy evaluation. Abbreviations: CAP, community-acquired pneumonia; HCAP, healthcare-associated pneumonia; ICD-9, International Classification of Diseases, Ninth Revision; VA, Veterans Affairs.

being frequently prescribed for longer than recommended, the reproducibility of this finding across different facilities has not been assessed. ${ }^{15,16}$ The ASTF collaborated with the VHA Center for Medication Safety to assess total duration of antimicrobial therapy prescribed for veterans hospitalized with uncomplicated pneumonia. ${ }^{17}$

\section{METHODS}

This retrospective multicenter evaluation was conducted in 30 VHA facilities that volunteered to participate in this project. Inpatients discharged with a primary International Classification of Diseases, Ninth Revision, Clinical Modification (ICD-9-CM) diagnosis code for pneumonia (or pneumonia diagnosis secondary to primary sepsis diagnosis) during 2013 were evaluated. ${ }^{18}$ Diagnoses, admissions, and patient demographics were identified using Veterans Affairs (VA) integrated databases through the Austin Integrated Technology Center. Up to 200 admissions per facility were randomly selected for review. Clinical pharmacists at each facility performed manual record reviews utilizing a standardized protocol and collection form. Completed cases were uploaded to a central database for analysis. Standardized chart abstraction was facilitated by detailed instructions, a data dictionary, and monthly conference calls.

Inclusion criteria required patient admission to any medical ward including intensive care unit (ICU) wards for $\geq 48$ hours, receipt of $>24$ hours inpatient antimicrobial therapy (eg, at least 2 doses of a oncedaily antibiotic), documentation of pneumonia discharge diagnosis, and survival until discharge. Exclusion criteria were: complicated pneumonia (lung abscess, necrotizing pneumonia, thoracentesis performed), significant immunosuppression (cancer chemotherapy or absolute neutrophil count $<1500$ cell/ $\mathrm{mm}^{3}$ within 28 days, organ transplantation, human immunodeficiency virus infection); or extrapulmonary infection (eg, meningitis, endocarditis). ${ }^{3}$ Patients were also excluded if directly transferred from another inpatient facility, pneumonia occurred $>48$ hours after admission, index hospitalization was $>14$ days, previously hospitalized within 28 days prior to index admission, or discharged without documentation of completing a full course of therapy. In addition, patients who received initial therapy discordant with culture and susceptibility findings, were not clinically stable by discharge, or had gram-negative nonfermentative bacilli cultured were excluded from analysis because according to the guidelines, either data are 


\begin{tabular}{|c|c|}
\hline Characteristic & Value \\
\hline Age, y, mean \pm SD & $71.8(12.7)$ \\
\hline Gender, male, n (\%) & $1,680(96.6)$ \\
\hline \multicolumn{2}{|l|}{ Living environment at time of index admission, $n(\%)$} \\
\hline Home & $1,416(81.4)$ \\
\hline VA community-based living center & $88(5.1)$ \\
\hline Non-VA long-term skilled care facility & $95(5.5)$ \\
\hline Assisted living facility & $52(2.9)$ \\
\hline Not documented & $46(2.7)$ \\
\hline Other & $29(1.7)$ \\
\hline \multicolumn{2}{|l|}{ Prior healthcare exposures, $n(\%)$} \\
\hline Prior hospitalization within last 90 days & $310(17.8)$ \\
\hline Residence in a long-term skilled care facility in last 90 days & $209(12.0)$ \\
\hline Chronic dialysis within last 28 days & $52(3.0)$ \\
\hline Intravenous antimicrobials within last 28 days & $76(4.4)$ \\
\hline Wound, tracheostomy, or ventilator care in last 28 days & $37(2.1)$ \\
\hline Community-acquired pneumonia, $n(\%)$ & $1,195(68.7)$ \\
\hline Healthcare-associated pneumonia, $n(\%)$ & $544(31.3)$ \\
\hline \multicolumn{2}{|l|}{ Comorbidities, n (\%) } \\
\hline Renal disease & 438 (25.2) \\
\hline Liver disease & $39(2.2)$ \\
\hline Congestive heart failure & $436(25.1)$ \\
\hline Cerebrovascular disease & $356(20.4)$ \\
\hline Neoplastic disease (excluding skin) & $384(22.1)$ \\
\hline \multicolumn{2}{|l|}{ Severity of illness, $n(\%)$} \\
\hline \multicolumn{2}{|l|}{ Pneumonia Severity Index } \\
\hline Class I & $30(1.8)$ \\
\hline Class II & $198(11.4)$ \\
\hline Class III & $349(20.1)$ \\
\hline Class IV & $759(43.6)$ \\
\hline Class V & $403(23.2)$ \\
\hline Intensive care upon admission & $212(12.2)$ \\
\hline Culture collection $\leq 48$ hours of admission, $n(\%)$ & $1,687(97.0)$ \\
\hline Blood & $1,631(96.7)$ \\
\hline Lower respiratory tract (sputum) & $673(39.9)$ \\
\hline Bronchoalveolar lavage & $20(1.2)$ \\
\hline Urine & $632(37.5)$ \\
\hline Skin/wound & $3(0.2)$ \\
\hline Other & $158(9.4)$ \\
\hline \multicolumn{2}{|l|}{ Facility complexity, n (\%) } \\
\hline Level 1a-c & $1,286(74.0)$ \\
\hline Level 2 & 437 (25.1) \\
\hline Level 3 & $16(0.9)$ \\
\hline
\end{tabular}

NOTE: Abbreviations: SD, standard deviation; VA, Veterans Affairs.

lacking to support a short duration of therapy such as initial discordant therapy, or a longer duration of therapy may be warranted such as gram-negative nonfermentative bacilli and clinical instability at discharge. ${ }^{4}$ Our intent for these exclusions was to minimize bias against clinician decision making for cases where a longer duration of therapy may have been appropriate.

Patients meeting all criteria had the following abstracted: demographics; prior healthcare exposures, admitting location (ICU or non-ICU ward), parameters for calculation of Pneumonia Severity Index (PSI), culture results obtained $\leq 48$ hours of admission, duration of antimicrobials administered during hospitalization and prescribed upon discharge (or recommendations for outpatient duration in the discharge summary for patients receiving medications from non-VA sources), daily clinical stability assessment, Clostridium difficile infection (CDI) test results, and readmission or death within 28 days of discharge. ${ }^{19}$

Guideline-similar CAP therapy duration was defined as a minimum of 5 days of antimicrobials, up to a maximum of 3 additional days beginning the first day the patient was afebrile and exhibited $\leq 1$ sign of clinical instability (heart rate $>100$ beats/minute, respiratory rate $>24$ breaths/minute, systolic blood pressure $<90$, oxygen saturation $<90 \%$ or partial pressure of oxygen $<60 \mathrm{~mm} \mathrm{Hg}$ on room air or baseline $\mathrm{O}_{2}$ requirements, or not returned to baseline mental status). ${ }^{3}$ This definition was made by consensus decision of the investigators and was necessary to operationalize the relationship between clinical stability and appropriate duration of therapy. Guideline-similar HCAP therapy duration was defined as 8 days. ${ }^{4} \mathrm{CDI}$ was defined in accordance with VA criteria for hospital onset and community-onset healthcare-facilityassociated CDI. ${ }^{20}$ All-cause hospital readmission and all-cause death were defined as inpatient readmission or any death, respectively, within 28 days after discharge for the pneumonia admission.

Demographics, comorbidities, microbiology results, antimicrobial utilization, CDI, readmission, and death rates between guideline-similar and guideline-excessive duration of antimicrobial therapy groups were characterized with descriptive statistics, Mann-Whitney $U$ test, or $\chi^{2}$ test as indicated (significance defined as $P$ $<0.05$ ). Multivariable logistic regression (SAS version 9.3 [SAS Institute, Cary, NC]) was used to assess association between duration of therapy exceeding recommended guidelines with all-cause readmission and all-cause death after adjustment for pertinent covariates. Odds ratios (OR) with 95\% confidence intervals $( \pm 95 \% \mathrm{CI})$ were reported. This medication utilization evaluation (MUE) was reviewed by the Hines VHA Institutional Review Board for Human Subjects Protection. Based on VHA Policy Handbook 1058.05, which defines operations activities that may constitute research, the board determined that the evaluation constituted quality improvement rather than research, and thus was exempt from VHA Human Subjects Research requirements.

\section{RESULTS}

There were 3881 admissions eligible for chart review. After manual chart review of inclusion and exclusion criteria, $1739(44.8 \%)$ patients were available for duration of therapy analysis. (Figure 1). Only 1 admission for each patient was analyzed.

The cohort was comprised primarily of elderly male patients $(96.6 \%)$ of whom more than two-thirds were hospitalized for CAP (Table 1). Most patients had significant disease severity as indicated by PSI score; however, only $12 \%$ were directly admitted to the ICU. 
TABLE 2. Antimicrobials Administered During Hospitalization and Dispensed Upon Discharge

\begin{tabular}{|c|c|c|c|c|}
\hline \multicolumn{4}{|c|}{ Inpatient Antimicrobials Administered* } & \multirow[b]{2}{*}{ Significance } \\
\hline & $\begin{array}{l}\text { Portion of Cohort Receiving } \\
\text { Antimicrobial, } n(\%), n=1,739\end{array}$ & $\begin{array}{c}\text { Therapy Duration } \\
\text { Similar With Guidelines, } n(\%), n=241\end{array}$ & $\begin{array}{l}\text { Therapy Duration Exceeding } \\
\text { Guidelines, } n(\%), n=1,498\end{array}$ & \\
\hline Third-generation cephalosporins & $809(46.5)$ & $75(31.1)$ & $734(49.0)$ & $<0.001 \dagger$ \\
\hline Fluoroquinolones & $836(48.1)$ & $114(47.3)$ & 722 (48.2) & 0.80 \\
\hline Macrolides & $788(45.3)$ & $90(37.3)$ & $698(46.6)$ & $<0.01 \dagger$ \\
\hline Pseudomonal $\beta$-lactams & $692(39.8)$ & $138(57.3)$ & $554(37.0)$ & $0.01 \ddagger$ \\
\hline Anti-MRSA antimicrobials & $663(38.1)$ & $135(56.0)$ & $528(35.3)$ & $<0.01 \ddagger$ \\
\hline Other $\beta$-lactams & $139(8.0)$ & $10(4.2)$ & $129(8.6)$ & 0.02 \\
\hline Tetracyclines & $119(6.8)$ & $14(5.8)$ & $105(7.0)$ & 0.49 \\
\hline Other & $97(5.6)$ & $15(6.2)$ & $82(5.5)$ & 0.64 \\
\hline
\end{tabular}

\begin{tabular}{|c|c|c|c|c|}
\hline \multicolumn{4}{|c|}{ Antimicrobials Dispensed or Recommended at Discharge§† } & \multirow[b]{2}{*}{ Significance } \\
\hline & $\begin{array}{c}\text { Portion of Cohort Receiving } \\
\text { Antimicrobial, } n(\%), n=1,471\end{array}$ & $\begin{array}{c}\text { Therapy Duration } \\
\text { Similar With Guidelines, } n(\%), n=151\end{array}$ & $\begin{array}{l}\text { Therapy Duration Exceeding } \\
\text { Guidelines, } n(\%), n=1,320\end{array}$ & \\
\hline Third-generation cephalosporins & $285(19.4)$ & $27(17.9)$ & $258(19.6)$ & 0.62 \\
\hline Fluoroquinolones & $813(55.3)$ & $95(62.9)$ & $718(54.4)$ & 0.05 \\
\hline Macrolides & $203(13.8)$ & $20(13.3)$ & $183(13.9)$ & 0.83 \\
\hline Pseudomonal $\beta$-lactams & $31(2.1)$ & $4(2.7)$ & $27(2.1)$ & 0.62 \\
\hline Anti-MRSA antimicrobials & $45(3.1)$ & $6(4.0)$ & $39(3.0)$ & 0.49 \\
\hline Other $\beta$-lactams & $239(16.3)$ & $13(8.6)$ & $226(17.1)$ & 0.01 \\
\hline Tetracyclines & $95(6.5)$ & $10(6.6)$ & $85(6.4)$ & 0.93 \\
\hline Other & $44(3.0)$ & $5(3.3)$ & $39(3.0)$ & 0.81 \\
\hline
\end{tabular}

NOTE: Third-generation cephalosporins: ceftriaxone, cefotaxime, cefpodoxime, cefprozil, cefdinir, cefuroxime. Fluoroquinolones: moxifloxacin, levofloxacin, ciprofloxacin. Macrolides: azithromycin, clarithromycin. Pseudomonal $\beta$-lactams: piperacillin/tazobactam, cefepime, ceftazidime, aztreonam, meropenem, imipenem. Anti-MRSA antimicrobials: vancomycin, linezolid, ceftaroline. Other $\beta$-lactams: ampicillin/sulbactam, amoxicillin/clavulanic acid, ampicillin, amoxicillin, penicillin, nafcillin, dicloxacillin, cefazolin, cephalexin, ertapenem. Tetracyclines: doxycycline, minocycline, tigecycline. Other: clindamycin, metronidazole, trimethoprim/sulfamethoxazole, gentamicin, tobramycin, amikacin, polymyxin B. Abbreviations: CAP, community-acquired pneumonia; HCAP, healthcare-associated pneumonia; MRSA, methicillin-resistant Staphylococcus aureus; VA, Veterans Affairs. *Includes all patients ( $\mathrm{n}$ = 1,739) administered at least 1 dose of antimicrobial. †The majority of patients in this group were CAP patients for whom the guideline-similar duration of therapy was less than that allowed for HCAP patients. †Note: The majority of patients in this group were HCAP patients for whom the guideline-similar duration of therapy was shorter than that allowed for CAP patients. §Includes all patients who had a VA prescription dispensed within 24 hours of hospital discharge or had an antimicrobial and duration recommended in the discharge summary.

Blood cultures were collected in $>95 \%$ of cases; lower respiratory cultures were obtained in $39.9 \%$ of cases.

Commonly administered antimicrobials during hospitalization and at discharge are summarized in Table 2. Anti-pseudomonal $\beta$-lactams and antimethicillin-resistant Staphylococcus aureus antimicrobials were more frequently administered to patients with HCAP, whereas third-generation cephalosporins and macrolides were more likely to be administered to patients with CAP. Fluoroquinolones were prescribed to $55.3 \%$ of patients upon discharge.

Overall, $13.9 \%$ of patients with uncomplicated pneumonia received guideline-similar duration of therapy (Table 3). A greater proportion of HCAP patients $(29.0 \%)$ received guideline-similar therapy duration as compared to CAP patients $(6.9 \%)(P<0.01$ (Table 3). Median duration of therapy was 7 days (interquartile range $[\mathrm{IQR}]=7-8$ days) for guideline-similar therapy compared to 10 days (9-13 days) for therapy duration in excess of guideline recommendations. Overall, 97.1 $\%$ of patients met clinical stability criteria before day 4 of therapy, yet $50 \%$ received $\geq 4$ days of intravenous (IV) therapy (median was 4 days, IQR $=3-6$ days). Antimicrobial therapy was generally completed after discharge, as only $17.3 \%$ received their entire treatment course during hospitalization. Median duration of outpatient oral (PO) antimicrobial therapy was twice as long for guideline-excessive therapy compared to guideline-similar therapy (6 vs 3 days), whereas duration of inpatient IV and PO antimicrobial therapy was similar. Patients discharged on a fluoroquinolone were more likely to receive guideline-similar duration of therapy. The VHA classifies facilities into 3 levels of complexity, with lower scores indicating more complex facilities. ${ }^{21}$ Guideline-similar therapy duration occurred in $10.4 \%$ of cases in lower complexity facilities (levels 2 and 3 ), and $15.1 \%$ in more complex facilities (level 1) $(P$ $=0.01)$. The median duration of therapy was similar for more and less complex facilities, respectively (10 days, IQR $=8-12$ days vs 10 days, IQR $=8-13$ days).

The 28-day postdischarge all-cause readmission rate for patients who received guideline-similar therapy duration was higher $(17.4 \%)$ than for patients who received therapy duration in excess of guideline recommendations $(12.2 \%)(P=0.03)$. After adjustment for covariates associated with readmission (HCAP, age, prior skilled nursing facility residence, PSI score comorbidity elements), we found no evidence that patients who received guideline-similar therapy duration were more likely to be readmitted than were patients who received guideline-excessive duration (OR: 1.1 [95\% CI: 0.8, 1.7]) (Table 3). Likewise, no difference in 28-day 


\begin{tabular}{|c|c|c|c|}
\hline Outcome & $\begin{array}{c}\text { Therapy Duration } \\
\text { Similar With IDSA/ATS Guidelines }\end{array}$ & $\begin{array}{l}\text { Therapy Duration in Excess of IDSA/ATS } \\
\text { Guideline Recommendations }\end{array}$ & Significance \\
\hline $\begin{array}{l}\text { Antimicrobial duration consistent with guideline rec- } \\
\text { ommendations, } n(\%)\end{array}$ & $241(13.9)$ & $1,498(86.1)$ & NR \\
\hline CAP*广 & $83(6.9)$ & $1,112(93.1)$ & NR \\
\hline HCAP*广 & $158(29.0)$ & $386(71.0)$ & NR \\
\hline Total days of therapy for pneumonia, median (IQR) & $7(7-8)$ & $10(9-13)$ & NR \\
\hline CAP & $6(5-9)$ & $10(8-12)$ & $<0.01$ \\
\hline HCAP & $7(7-8)$ & $11(10-14)$ & $<0.01$ \\
\hline $\begin{array}{l}\text { Days of IV therapy administered for pneumonia, } \\
\text { median (IQR) }\end{array}$ & $4(3-7)$ & $4(3-6)$ & 0.50 \\
\hline $\begin{array}{l}\text { Days of PO inpatient therapy administered, median } \\
\text { (IQR) }\end{array}$ & $1(0-3)$ & $1(0-3)$ & 0.78 \\
\hline $\begin{array}{l}\text { Days of P0 outpatient therapy dispensed at dis- } \\
\text { charge, median (IQR) }\end{array}$ & $3(2-5)$ & $6(4-7)$ & $<0.01$ \\
\hline $\begin{array}{l}\text { Days of PO outpatient therapy recommended in Dis- } \\
\text { charge Summary for patients without a VA pre- } \\
\text { scription, median (IQR)§ }\end{array}$ & $3(2-4)$ & $5(4-7)$ & $<0.01$ \\
\hline Aggregate 28-day hospital readmission, n (\%) & $42(17.4)$ & $183(12.2)$ & 0.03 \\
\hline CAP $\|$ 聿 & $7(8.4)$ & $112(10.1)$ & 0.58 \\
\hline HCAP $\| \#$ & $35(22.2)$ & $71(18.4)$ & 0.28 \\
\hline Aggregate 28-day CDI rate, n (\%) & $6(2.5)$ & $9(0.6)$ & 0.03 \\
\hline CAP $\|^{* *}$ & $1(1.2)$ & $6(0.5)$ & 0.44 \\
\hline HCAP $\|^{\| *}$ & $5(3.2)$ & $3(0.8)$ & 0.04 \\
\hline Aggregate 28-day death after discharge, n (\%) & $6(2.5)$ & $52(3.5)$ & 0.43 \\
\hline $\mathrm{CAP} \|^{* *}$ & $1(1.2)$ & $33(3.0)$ & 0.35 \\
\hline $\mathrm{HCAP} \|^{* *}$ & $5(3.2)$ & $19(4.9)$ & 0.37 \\
\hline
\end{tabular}

NOTE: Abbreviations: CAP, community acquired pneumonia; HCAP, healthcare-associated pneumonia; NR, not relevant. *Denominators for each row are stratified by all included and nonexcluded patients who had CAP and HCAP, respectively. $\mathrm{CCAP}$ versus HCAP, $P<0.01$. $\neq n=1,403 . \S n=76$. $\mid$ Denominators for each row are stratified by guideline concordance and discordance and patients who had CAP and $\mathrm{HCAP}$, respectively. $\uparrow$ Twenty-eightday hospital readmission, guideline concordant therapy, CAP versus HCAP, $P<0.01$. \# Twenty-eight-day hospital readmission, guideline discordant therapy, CAP versus HCAP, $P<0.01$. ${ }^{*} \mathrm{HCAP}$ versus CAP $P$ values not significant.

TABLE 4. Multivariable Models for 28-Day Readmission and Mortality

\begin{tabular}{lccc}
\hline Model Variables & $\begin{array}{c}\text { Odds } \\
\text { Ratio }\end{array}$ & $\begin{array}{c} \pm 95 \% \text { Confidence } \\
\text { Interval }\end{array}$ & $P$ Value \\
\hline Readmission model & & & \\
Duration of antibiotics & 1.11 & $0.75,1.64$ & 0.62 \\
HCAP & 1.94 & $1.38,2.72$ & $<0.01$ \\
Age & 1.01 & $1.00,1.03$ & 0.04 \\
Prior skilled nursing facility residence & 0.91 & $0.59,1.40$ & 0.67 \\
PSI score comorbidity elements & - & - & - \\
Neoplastic disease & 1.20 & $0.86,1.67$ & 0.29 \\
Liver disease & 1.55 & $0.66,3.64$ & 0.31 \\
CHF & 1.15 & $0.83,1.59$ & 0.41 \\
Cerebrovascular disease & 1.06 & $0.75,1.50$ & 0.75 \\
Renal disease & 1.51 & $1.09,2.08$ & 0.01 \\
Mortality model & & & \\
Duration of antibiotics & 0.53 & $0.23,1.22$ & 0.14 \\
HCAP & 2.53 & $1.38,4.65$ & $<0.01$ \\
Age & 1.06 & $1.03,1.09$ & $<0.01$ \\
Prior skilled nursing facility residence & 0.79 & $0.38,1.66$ & 0.53 \\
PSI score comorbidity elements & - & - & - \\
Neoplastic disease & 1.03 & $0.57,1.87$ & 0.91 \\
Liver disease & $<0.001$ & $<0.001,>999.9$ & 0.98 \\
CHF & 0.73 & $0.39,1.38$ & 0.34 \\
Cerebrovascular disease & 0.82 & $0.43,1.56$ & 0.55 \\
Renal disease & 0.72 & $0.39,1.35$ & 0.31 \\
\hline
\end{tabular}

NOTE: Abbreviations: CHF, congestive heart failure; HCAP, healthcare-associated pneumonia; PSI, Pneumonia Severity Index. all-cause postdischarge mortality was identified between guideline-similar and guideline-excessive duration after adjustment for the same covariates (adjusted OR: 0.5 [95\% CI: 0.2, 1.2]) (Table 4).

CDI cases $(n=15)$ were too sparse to adequately perform multivariable logistic regression analysis; however, a higher percentage of patients who received guideline-similar duration of therapy developed CDI compared to patients who received guideline-excessive duration of therapy $(40.0 \%$ vs $13.6 \%, P<0.01)$. The median duration of therapy for patients who did and did not develop CDI was similar ( 8 days, IQR $=7$ 14 days vs 10 days, IQR $=8-12$ days, $P=0.85$, respectively). Patients who developed CDI had a higher rate of HCAP diagnosis $(1.5 \%$ vs $0.6 \% ; P=$ 0.06 ), were more likely to have concomitant nonpneumonia infection $(40.0 \%$ vs $9.5 \%, P<0.01)$, have chronic comorbidity $(86.7 \%$ vs $59.1 \%, P=$ $0.03)$, and to have been admitted to the ICU $(26.7 \%$ vs $12.1 \%, P=0.09$ ).

\section{DISCUSSION}

IDSA/ATS guidelines for pneumonia duration of therapy generally agree with other professional society guidelines including the British Thoracic Society and National Institute for Health and Care Excellence. ${ }^{22,23}$ 
In contrast to existing evidence and guideline recommendations, this multi-centered evaluation identified prolonged durations of antimicrobial therapy prescribed in $93 \%$ and $71 \%$ of patients with uncomplicated CAP and HCAP (Table 3), respectively. ${ }^{3-12}$ Almost all $(97.1 \%)$ uncomplicated CAP and HCAP patients met clinical stability criteria before day 4 of hospitalization, yet the median duration of IV therapy was 4 days. Because criteria for IV to PO conversion and the clinical stability definition utilized in this analysis were similar, many patients may have been eligible for PO therapy earlier, favorably impacting length of stay, cost, and adverse effects. ${ }^{3,12,24-26}$ Although median days of inpatient PO therapy administered was 1 day (IQR $=0-3$ days), inpatient observation after $\mathrm{PO}$ conversion may not be necessary. The duration of PO therapy was based on calendar days, where if a patient received 1 dose of a once daily antibiotic (ie, levofloxacin), they were considered to have received 1 day of inpatient $\mathrm{PO}$ antibiotics even if discharged the same day.

Approximately half of all days of therapy occurred after discharge. Although the median therapy duration for inpatients was similar, the median duration of antimicrobials administered upon hospital discharge was twice as long for patients receiving guidelineexcessive compared to guideline-similar duration of therapy. The median excess in antibiotic duration is almost entirely accounted for by excess outpatient days of therapy. This is an important consideration for antimicrobial stewardship programs that tend to focus on inpatient antimicrobial use.

Noteworthy observations include the low rate of respiratory tract culture collection $(41 \%)$ and frequent use of fluoroquinolones upon discharge. Collection of respiratory tract cultures is recommended for all patients with HCAP and patients with CAP who have risk factors for resistant pathogens, characteristics that were common in this cohort. ${ }^{3,4}$ Recently, we identified that respiratory culture collection is associated with increased de-escalation rates in HCAP, and that culture-negative patients frequently receive fluoroquinolones. ${ }^{27}$ IDSA/ATS CAP guidelines discourage empirically switching to PO fluoroquinolone therapy based on bioavailability considerations alone. ${ }^{3}$ Further, fluoroquinolones are considered to be associated with high risk of CDI. ${ }^{28,29}$ Prescription of fluoroquinolone upon discharge was associated with guidelinesimilar duration of therapy and was not shown to be associated with CDI; however, power to detect differences between exposures to specific antimicrobials and CDI was low.

CDI was more common in patients with CAP $(1.2 \%$ vs $0.5 \%)$ and $\operatorname{HCAP}(3.2 \%$ vs $0.8 \%)$ who received duration of therapy similar with guideline recommendations. This observation is confounded, as patients with CDI had significantly greater comorbidity as well as secondary infections and tended to more frequently receive ICU care. There were no differences in adjusted rates of readmission or death between patients receiving guideline-similar and guidelineexcessive duration of therapy.

Evaluation strengths included exclusion of patients with complicating conditions possibly requiring prolonged antimicrobial treatment courses, which allowed the evaluation to focus on patients most likely to benefit from shorter course therapy. The definition of appropriate therapy duration was based upon daily assessment of clinical stability criteria that paralleled the CAP guidelines. The definition utilized objective parameters while accounting for patient variability in achieving clinical stability criteria. Finally, the analyses of clinical end points suggest that shorter duration of therapy may be as safe and effective as longer duration of therapy in uncomplicated pneumonia.

Limitations include those common to other analyses conducted within the VHA, including a predominantly elderly male cohort. ${ }^{30}$ Only ICD-9-CM codes consistent with a discharge diagnosis of pneumonia were used to identify the cohort, and clinical impressions not documented in the medical record may have impacted the clinician's treatment duration decisions. The upper limit of appropriate duration of therapy for CAP was arbitrarily set at up to 3 days beyond meeting clinical stability criteria to provide a reasonable duration of appropriate therapy beyond clinical stability to operationalize the duration of therapy recommendations within the context of the IDSA/ATS guidelines. Additionally, CIs for the ORs of readmission and mortality were broad, and thus too imprecise to determine whether guideline-similar durations increased or decreased readmission or mortality in comparison with therapy that exceeded guideline recommendations. We could not fully assess the potential for association between guideline-excessive therapy duration and risk for CDI due to sparse cases. Finally, non-VA prescription data were not available for all patients, and we relied on intended duration of therapy as recommended by the discharging provider in $4.1 \%$ of cases.

Most quality assessments of pneumonia treatment have focused on antimicrobial selection and timely administration or conversion from IV to PO therapy. ${ }^{31,32}$ This evaluation identified potential opportunities for expansion of antimicrobial stewardship activities during the transition of care setting. The efficacy of short-course $\beta$-lactam, macrolide, or fluoroquinolone therapy for CAP appears equivalent to longer treatment regimens with no difference in adverse event rates, suggesting that optimal duration of therapy may be a rational target for quality improvement. ${ }^{5-12,15,31}$ Recommendations for HCAP duration of therapy are extrapolated from a prospective multicentered study, which randomized patients with hospital-acquired pneumonia to receive 8 versus 
15 days of therapy, that identified similar outcomes to ours. $^{4,12}$

Single-center studies have identified that antimicrobial therapy for pneumonia is frequently prescribed for longer than recommended by guidelines, which found a similar median duration of therapy as our evaluation. ${ }^{15,16}$ Similar to Jenkins et al., we observed a high rate of fluoroquinolone prescriptions upon discharge. ${ }^{16}$

There are few published examples of interventions designed to limit excessive duration of therapy, particularly for antimicrobials prescribed upon hospital discharge. ${ }^{15,33,34}$ Serial procalcitonin measurements have been used to guide duration of therapy for pneumonia; however, the cost-benefit ratio of procalcitonin measurement is unclear. ${ }^{35,36}$ Procalcitonin use was uncommon, and none of the participating facilities in our evaluation utilized a specific algorithm to guide therapy duration. Limited data suggest that patientlevel prospective audit with feedback may be effective. Advic et al. evaluated management of presumed CAP before and after education and prospective feedback to medical teams concerning antimicrobial selection and duration of therapy. ${ }^{15}$ The intervention led to a decrease in median duration of therapy from 10 days $(\mathrm{IQR}=8-13$ days) to 7 days (IQR $=7-8$ days) without increasing clinical failure or readmission rates. We recently reported a single-center evaluation in which pharmacists utilizing a decision support tool while performing discharge medication reconciliation were able to reduce excessive mean duration of therapy from 9.5 days ( \pm 2.4 days) to 8.3 days ( \pm 2.9 days) in patients without complicated pneumonia, with a $19.2 \%$ reduction in duration of therapy prescribed at discharge. ${ }^{37}$ A similar approach utilizing pharmacists performing discharge review has recently been reported in a community hospital. ${ }^{38}$

Future work should recognize that few patients complete their entire course of therapy as inpatients, and the majority of treatment is prescribed upon discharge. Pivotal time points for antimicrobial stewardship intervention include day 2 to 3 of hospitalization when conveying suggestions for antimicrobial deescalation and/or IV to PO conversion, and toward the end of hospitalization during discharge planning. Although it may not be feasible for antimicrobial stewards to review all uncomplicated cases of pneumonia during hospitalization, most facilities have a systematic process for reviewing medications during transitions of care. We believe that interventions intended to assess and recommend shortened courses of therapy are appropriate. These interventions should include a mechanism for support by stewardship personnel or other infectious diseases specialists. Based on our evaluation, the ASTF produced and disseminated clinical guidance documents and tools to triage pneumonia case severity and assess response to therapy. Qualified personnel are encouraged to use this information to make recommendations to providers regarding excessive duration of therapy for uncomplicated cases where appropriate. Other work should include an in-depth assessment of clinical outcomes related to treatment duration, investigation of provider rationale for prolonged treatment, and duration of antimicrobial therapy prescribed upon discharge for other common disease states. Finally, manual chart review to classify uncomplicated cases and related outcomes was laborious, and automated case identification is technologically plausible and should be explored. ${ }^{39}$

In conclusion, this national VHA MUE found that patients with uncomplicated pneumonia were commonly prescribed antimicrobials for the duration of therapy in excess of guideline recommendations. Patients with uncomplicated pneumonia who received therapy duration consistent with guideline recommendations did not have significantly different all-cause readmission and death rates compared to patients receiving prolonged treatment. Approximately half of all therapy was prescribed upon hospital discharge, and clinicians as well as antimicrobial stewardship programs should consider these findings to address excessive duration of antimicrobial therapy upon hospital discharge.

\section{Acknowledgements}

The authors acknowledge Dr. Michael Fine for his assistance with utilization of the Pneumonia Severity Index, Kenneth Bukowski for assisting with development of data collection tools and data management, and members of the Antimicrobial Stewardship Taskforce Implementation Sub-Committee. Collaborators in the Pneumonia Duration of Therapy Medication Utilization Evaluation Group include: Biloxi VA (VA Gulf Coast): Cheryl Hankins, PharmD, BCPS; Central Alabama VAMC: Lauren Rass, PharmD, BCPS, Kelly Mooney, PharmD, BCPS; Central Arkansas: Nicholas Tinsley, MS, PharmD; Chillicothe VA: Stephen Hanson, PharmD, BCPS, Beth Gallaugher, BSN, RN, Elizabeth Baltenberger, PharmD; Cincinnati VA: Jason Hiett, PharmD, BCPS, Victoria Tate, PharmD, BCPS, Brian Salzman, PharmD; Dorn Medical Center: MaryAnne Maurer, PharmD, BCPS, BCACP, Rebekah Sipes, PharmD, BCACP, Ginger Ervin, PharmD; Dwight D. Eisenhower VAMC: Emily Potter, PharmD; Hudson Valley: Rita Lee Bodine, PharmD, Clement Chen, PharmD, Cristina Fantino, PharmD; James H. Quillen VAMC: Marty Vannoy, PharmD, BCPS, Erin Harshbarger, PharmD, Kristen Nelsen, PharmD; Jesse Brown VAMC: Lisa Young, PharmD, BCPS, AQID, Andrea Bidlencik, PharmD, BCPS; Kansas City VA: Jamie Guyear, PharmD, AQ-ID, Ann Ungerman, PharmD, BCPS, Lauri Witt, PharmD, BCACP; Louis Stokes Cleveland VAMC: Amy Hirsch, PharmD, BCPS, Steven Adoryan, PharmD, BCP-CC, Amanda Miller, PharmD, BCPS; Maine VAMC: Joel Coon, PharmD, Rachel Naida, PharmD, Kelly Grossman, PharmD; Martinsburg VAMC: Kelly Li, PharmD, Sarah Mickanis, PharmD, BCPS; Miami VA Medical Center: Mara Carrasquillo, BS, PharmD, Maribel Toro, PharmD; North Florida/South Georgia Veterans Health System: Nora Morgan, PharmD, Hugh Frank, PharmD, BCPS, BCPP, Sarah Onofrio, PharmD, BCPS; North Texas HCS: Susan Duquaine, PharmD, BCPS, AQ-ID, Ruben Villaneuva, PharmD, BCPS, Jaela Dahl, PharmD, BCPS; Ozarks: Andrew Siler, PharmD, BCPS, Michele Walker, PharmD, CGP, Jennifer Cole, PharmD, BCPS, BCCCP; Providence VAMC: Kerry LaPlante, PharmD, FCCP, Lindsey Williamson, PharmD; Richmond VA: Daniel Tassone, PharmD, BCPS; Salisbury VAMC: Brett Norem, PharmD, Marrisa Ragonesi, PharmD; San Juan VA: Monica Sanabria-Seda, PharmD, BCPS, Jaime Velez-Fores, PharmD, BCPS, AQ-ID, Norma Ayala-Burgos, PharmD; Sioux Falls VA: Andrea Aylward, PharmD, BCPS; South Texas HCS: Kelly Echevarria, PharmD, BCPS, AQ-ID, Manuel Escobar, PharmD; Tennessee Valley HCS: Casey Ryals, PharmD, BCACP, Molly Hurst, PharmD, Jonathan Hale, PharmD; VA Central Iowa Health Care System: Jenny Phabmixay, PharmD, BCPS, Mackenzie Brown, PharmD, BCPS, Cynthia Muthusi, PharmD, BCPS; VA Loma Linda: Tony Chau, 
PharmD; VA Sierra Nevada: Scott Mambourg, PharmD, BCPS, AAHIVP, Matthew Han, PharmD, Nathan Mihoch, PharmD; VA WNY Healthcare System: Kari Mergenhagen, PharmD, BCPS, AQ-ID, Christine Ruh, PharmD, BCPS; Veterans Affairs Salt Lake City Health System: Emily Spivak, MD, MHS, Patricia Orlando, PharmD

Disclosures: Karl Madaras-Kelly is employed full time by Idaho State University and has a without compensation appointment as a clinical pharmacist at the Boise VA Medical Center. He receives grant support unrelated to this work through the Department of Veterans Affairs subcontracted to Idaho State University. Muriel Burk is employed full time through the Department of Veterans Affairs as clinical pharmacy specialist in outcomes and medication safety evaluation. Christina Caplinger was employed by the Department of Veterans Affairs as an infectious diseases fellow at the time this work was completed. She is currently employed by Micromedex. Jefferson Bohan is employed full time by the Department of Veterans Affairs as an infectious diseases fellow. Melinda Neuhauser is employed full time through the Department of Veterans Affairs as a clinical pharmacy specialist-infectious diseases. Matthew Goetz is employed full time through the Department of Veterans Affairs as an infectious diseases physician. Rhongping Zhang is employed full time through the Department of Veterans Affairs as a data analyst. Francesca Cunningham is employed full time through the Department of Veterans Affairs as the director of the VA Center for Medication Safety. This work was supported with resources and use of the Department of Veterans Affairs healthcare system. The views expressed in this article are solely those of the authors and do not necessarily reflect the position or policy of the Department of Veterans Affairs. The authors report no conflicts of interest.

\section{References}

1. Centers for Disease Control and Prevention. National hospital discharge survey 2010. Available at: http://www.cdc.gov/nchs/fastats/ pneumonia.htm. Accessed December 1, 2014.

2. Barlam TF, Cosgrove SE, Abbo LM, et al. Implementing an antibiotic stewardship program: guidelines by the Infectious Diseases Society of America and the Society for Healthcare Epidemiology of America. Clin Infect Dis. 2016;62(10):e51-e77.

3. Mandell LA, Wunderink RG, Anzueto A, et al. Infectious Diseases Society of America/American Thoracic Society consensus guidelines on the management of community-acquired pneumonia in adults. Clin Infect Dis. 2007;44(suppl 2):S27-S72.

4. American Thoracic Society; Infectious Diseases Society of America. Guidelines for the management of adults with hospital-acquired, ventilator-associated, and healthcare-associated pneumonia. Am J Respir Crit Care Med. 2005;171(4):388-416.

5. Dimopoulos G, Matthaiou DK, Karageorgopoulos DE, et al. Shortversus long-course antibacterial therapy for community-acquired pneumonia: a meta-analysis. Drugs. 2008;68(13):1841-1854.

6. Li JZ, Winston LG, Moore DH, et al. Efficacy of short-course antibiotic regimens for community-acquired pneumonia: a meta-analysis. Am J Med. 2007;120:783-790.

7. Dunbar LM, Wunderink RG, Habib MP, et al. High-dose, shortcourse levofloxacin for community-acquired pneumonia: a new treatment paradigm. Clin Infect Dis. 2003;37:752-760.

8. Siegel RE, Alicea M, Lee A, et al. Comparison of 7 versus 10 days of antibiotic therapy for hospitalized patients with uncomplicated community-acquired pneumonia: a prospective. Am J Ther. 1999; 6(4):217-222

9. el Moussaoui R, de Borgie CA, van den Broek P, et al. Effectiveness of discontinuing antibiotic treatment after three days versus eight days in mild to moderate-severe community acquired pneumonia: randomised, double blind trial. BMJ. 2006;332(7554):1355.

10. Rizzato G, Montemurro L, Fraioli P, et al. Efficacy of a three day course of azithromycin in moderately severe community-acquired pneumonia. Eur Respir J. 1995;8(3):398-402.

11. Chastre J, Wolff M, Fagon J, et al. Comparison of 8 vs 15 days of antibiotic therapy for ventilator-associated pneumonia in adults: a randomized trial. JAMA. 2003;290(19):2588-2598.

12. Oosterheert JJ, Bonten MJ, Schneider MM, et al. Effectiveness of early switch from intravenous to oral antibiotics in severe community acquired pneumonia: multicentre randomized trial. BMJ. 2006; 333(7580):1193

13. Graber CJ, Madaras-Kelly K, Jones MM, Neuhauser MM, Goetz MB. Unnecessary antimicrobial use in the context of Clostridium difficile infection: a call to arms for the Veterans Affairs Antimicrobial Stewardship Task Force. Infect Control Hosp Epidemiol. 2013;34(6):651653.

14. VHA Directive 1031. Antimicrobial stewardship programs. Available at: https://www1.va.gov/vhapublications/ViewPublication.asp?pub_ $\mathrm{ID}=2964$. Accessed December 1, 2014.

15. Advic E, Cushinotto LA, Hughes AH, et al. Impact of an antimicrobial stewardship intervention on shortening the duration of therapy for community-acquired pneumonia. Clin Infect Dis. 2012;54:15811587.

16. Jenkins TC, Stella SA, Cervantes L, et al. Targets for antibiotic and healthcare resource stewardship in inpatient community-acquired pneumonia: a comparison of management practices with National Guideline Recommendations. Infection. 2013;41(1):135-144.

17. Sales MM, Cunningham FE, Glassman PA, Valentino MA, Good CB. Pharmacy benefits management in the Veterans Health Administration: 1995 to 2003. Am J Manag Care. 2005;11(2):104-112.

18. Aronsky D, Haug PJ, Lagor C, Dean NC. Accuracy of administrative data for identifying patients with pneumonia. Am J Med Qual. 2005; 20(6):319-328.

19. Fine MJ, Auble TE, Yealy DM, et al. A prediction rule to identify lowrisk patients with community-acquired pneumonia. N Engl J Med. 1997;336:243-250

20. Evans ME, Simbartl LA, Kralovic SM, Jain R, Roselle GA. Clostridium difficile infections in Veterans Health Administration acute care facilities. Infect Control Hosp Epidemiol. 2014;35(8):1037-1042.

21. Korom-Djakovic D, Canamucio A, Lempa M, Yano EM, Long JA. Organization complexity and primary care providers' perceptions of quality improvement culture within the Veterans Health Administration. Am J Med Qual. 2016;31(2):139-146.

22. Lim WS, Baudouin SV, George RC, et al. BTS guidelines for the management of community acquired pneumonia in adults: update 2009. Thorax. 2009;64(suppl 3):iii1-iii55.

23. National Institute for Health and Care Excellence. Pneumonia in adults: diagnosis and management. Available at: http://www.nice.org. uk/guidance/cg191. Published December 2014. Accessed May 9, 2016.

24. Siegel RE, Halpern NA, Almenoff PL, Lee A, Cashin R, Greene JG. A prospective randomized study of inpatient IV antibiotics for community-acquired pneumonia: the optimal duration of therapy. Chest. 1996;110(4):965-971.

25. Ramirez JA, Vargas S, Ritter GW, et al. Early switch from intravenous to oral antibiotics and early hospital discharge: a prospective observational study of 200 consecutive patients with community-acquired pneumonia. Arch Intern Med. 1999;159(20):2449-2454.

26. Sallach-Ruma R, Nieman J, Sankaranarayanan J, Reardon T. Correlates and economic and clinical outcomes of an adult IV to PO antimicrobial conversion program at an academic medical center in Midwest United States. J Pharm Pract. 2015;28(3):238-248.

27. Madaras-Kelly K, Jones M, Remington R, et al. Antimicrobial Deescalation of treatment for healthcare-associated pneumonia within the Veterans Healthcare Administration. I Antimicrob Chemother. 2016;71(2):539-546.

28. Deshpande A, Pasupuleti V, Thota P, et al. Community-associated Clostridium difficile infection and antibiotics: a meta-analysis. I Antimicrob Chemother. 2013;68(9):1951.

29. Brown KA, Khanafer N, Daneman N, Fisman DN. Meta-analysis of antibiotics and the risk of community-associated Clostridium difficle infection. Antimicrob Agents Chemother. 2013;57(5):2326-2332.

30. Rosen AK, Loveland S, Anderson JJ, et al. Evaluating diagnosis-based case-mix measures: how well do they apply to the VA population? Med Care. 2001;39:692-704.

31. Nussenblatt V, Avdic E, Cosgrove S. What is the role of antimicrobial stewardship in improving outcomes of patients with CAP? Infect Dis Clin North Am. 2013;27(1):211-228.

32. Lee JS, Nsa W, Hausmann LR, et al. Quality of care for elderly patients hospitalized for pneumonia in the United States, 2006 to 2010. JAMA Intern Med. 2014;174(11):1806-1814.

33. Aldeyab MA, Kearney MP, Scott MG, et al. An evaluation of the impact of antibiotic stewardship on reducing the use of high-risk antibiotics and its effect on the incidence of Clostridium difficile infection in hospital settings. I Antimicrob Chemother. 2012;67(12):29882996

34. Fridkin S, Baggs J, Fagan R, et al.; Centers for Disease Control and Prevention. Vital signs: improving antibiotic use among hospitalized patients. MMWR Morb Mortal Wkly Rep. 2014;63(9):194-200.

35. Schuetz P, Christ-Crain M, Thomann R et al. Effect of procalcitoninbased guidelines vs standard guidelines on antibiotic use in lower respiratory tract infections: the ProHOSP randomized controlled trial. JAMA. 2009;302(10):1059-1066.

36. Smith KJ, Wateska A, Nowalk MP, et al. Cost-effectiveness of procalcitonin-guided antibiotic use in community acquired pneumonia. J Gen Intern Med. 2013;28(9):1157-1164.

37. Caplinger C, Crane K, Wilkin M, Bohan J, Remington R, MadarasKelly KJ. Interim evaluation of a Protocol to Optimize the Duration of Pneumonia Therapy at Hospital Discharge. Open Forum Infect Dis. 2015;2(suppl 1):S379.

38. Yogo N, Young H, Shihadeh $\mathrm{K}$, et al. Intervention to improve antibiotic selection and shorten treatment durations at the time of hospital discharge. Open Forum Infect Dis. 2015;2(suppl 1):S1.

39. DeLisle S, Kim B, Deepak J, et al. Using the electronic medical record to identify community-acquired pneumonia: toward a replicable automated strategy. PLoS One. 2013;8(8):e70944. 